\title{
Incidental Finding of the Sternalis Muscle on Chest Multidetector-row Computed Tomography (MDCT): The Diagnostic Value of Additional Postprocessed MDCT Images for an Uncommon Muscular Variant
}

\author{
Shigeru Kobayashi ${ }^{1}$, Hidenori Tomizawa ${ }^{2}$, Yuuko Tomizawa ${ }^{2}$ and Yuuichi Manaka ${ }^{2}$
}

\begin{abstract}
We herein report two cases of incidental finding of the sternalis muscle in the right anterior chest wall on multidetector-row computed tomography (MDCT). We emphasize the diagnostic utility and value of additional postprocessed MDCT images, which can be easily created using volume data sets of MDCT, in establishing an accurate diagnosis of the sternalis muscle, an uncommon muscular variant.
\end{abstract}

Key words: sternalis muscle, accessory muscle, normal variation, thoracic wall, multidetector-row computed tomography (MDCT), postprocessed image

(Intern Med 52: 1137-1139, 2013)

(DOI: 10.2169/internalmedicine.52.9392)

\section{Introduction}

The sternalis muscle is an uncommon anatomic variant of the anterior chest wall muscles. There are more than 20 terms used to describe this anatomic variant, including episternalis, presternalis, rectus thoracis, rectus sterni and superficial rectus abdominis (1). The etiology and function of the sternalis muscle have remained unclear until recently. This muscle runs from the infraclavicular region to approximately the caudal aspect of the sternum and is located subcutaneously over the pectoralis major with a course parallel to the sternum (2). With the rapid advancement of imaging technology, the sternalis muscle has recently become detected more regularly on thin-slice multidetector-row computed tomography (MDCT) images $(2,3)$. However, diagnosing the sternalis muscle remains challenging because this muscle can be easily overlooked or misdiagnosed as a malignant lesion on imaging examinations such as mammography and computed tomography (CT) not only as a result of its low incidence, but also as a result of medical professionals' unfamiliarity with it $(3,4)$. In contrast, modern MDCT scanners are able to obtain continuous volume data sets with thin-slice collimation. Therefore, fine postprocessed images such as two-dimensional multiplanar reconstruction (2DMPR) and three-dimensional volume rendered (3D-VR) reconstruction can be immediately and easily created. An interactive manipulation on a scanner console or workstation can simultaneously facilitate the construction of appropriate images, resulting in additional information that helps to establish an accurate diagnosis of normal variants as well as pathological conditions $(3,5,6)$. Accurately interpreting MDCT images obtained from multidirectional views is important for reducing the need to perform additional examinations or biopsies when uncommon normal variants such as the sternalis muscle are encountered incidentally (4). We herein present two cases of the sternalis muscle in the right anterior chest wall detected incidentally on chest MDCT examinations in which additional postprocessed MDCT images combined with routine axial images assisted in establishing an accurate diagnosis of this uncommon muscular variant.

\section{Case Reports}

Case 1 (Fig. 1) involved a 74-year-old woman who was prescribed medication for hypertension at our clinic. Although she had no pulmonary symptoms, the patient desired to undergo a screening chest MDCT examination. Case 2

${ }^{1}$ Department of Radiology, Haga Red Cross Hospital, Japan and ${ }^{2}$ Tomizawa Heart Clinic, Japan Received for publication November 26, 2012; Accepted for publication January 23, 2013 Correspondence to Dr. Shigeru Kobayashi, skoba@jichi.ac.jp 


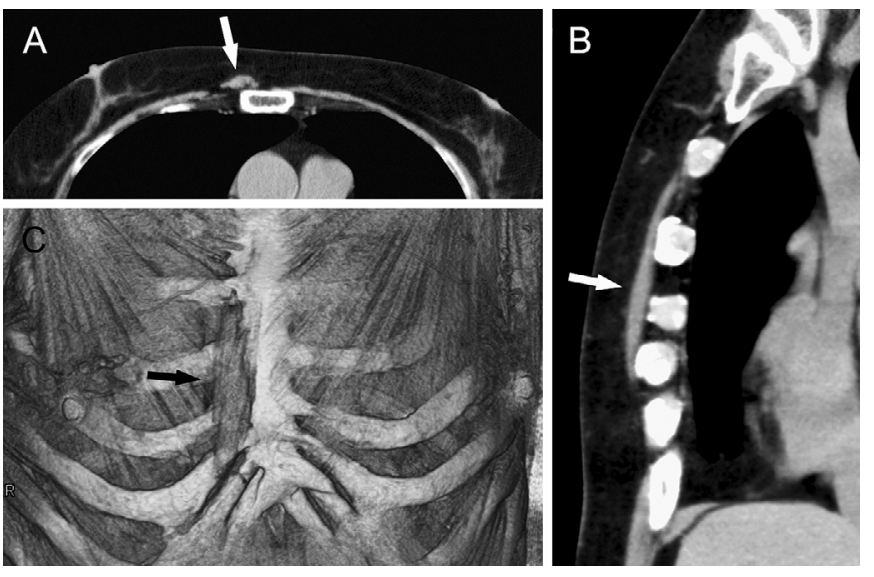

Figure 1. A 74-year-old woman with a sternalis muscle in the right anterior chest wall. A: A routine axial MDCT image shows a soft tissue mass (arrow), i.e., the sternalis muscle, which lies superficial to the right edge of the sternum. B: A postprocessed 2D-MPR image in the sagittal section reveals the corresponding soft tissue mass (arrow), which runs longitudinally in a craniocaudal direction. C: A 3D-VR image provides the entire configuration of the sternalis muscle, which has a fusiform shape with fascicle formation (arrow), thus reflecting a muscular architecture similar to that of other musculature in the chest wall.

(Fig. 2) involved a 63-year-old woman who was prescribed medication for hypertension and valvular heart disease at our clinic. The patient presented with prolonged coughing and sputum production following a high fever occurring one month previously. A chest MDCT examination was performed to evaluate the lungs, and active inflammatory changes were revealed in S6 of the left lower lobe.

In both cases, nonenhanced chest MDCT examinations were conducted using a 64-row MDCT scanner (Aquilion TSX-101A; Toshiba, Tochigi, Japan) during inspiratory breath-holding in the supine position. The scan parameters were as follows: tube voltage, $120 \mathrm{kV}$; tube current, auto $\mathrm{mA}$; collimation, $32 \times 0.5 \mathrm{~mm}$; pitch factor, 1.172 ; and rotation speed, $0.5 \mathrm{~s}$. From each source CT data set, routine axial MDCT images were reconstructed with a 5-mm slice thickness and a 5-mm interval on a CT console. For these cases, postprocessed 2D-MPR images of the sagittal and coronal sections were additionally reconstructed with a 2$\mathrm{mm}$ slice thickness and a 2-mm interval. 3D-VR images were also simultaneously created on a workstation (Ziostation version 2.0.5.1; Ziosoft Inc., Tokyo, Japan) using the same volume data set.

While the radiologist evaluated both cases on routine axial MDCT images, soft tissue masses were detected incidentally in the right anterior chest wall in both patients. There were no clinical symptoms related to the masses. For further exploration, additional postprocessed MDCT images, including sagittal and coronal 2D-MPR, and 3D-VR reconstruction were immediately created from the source CT data sets. On sagittal 2D-MPR images, each mass in the right anterior chest wall appeared flattened and ran craniocaudally. On

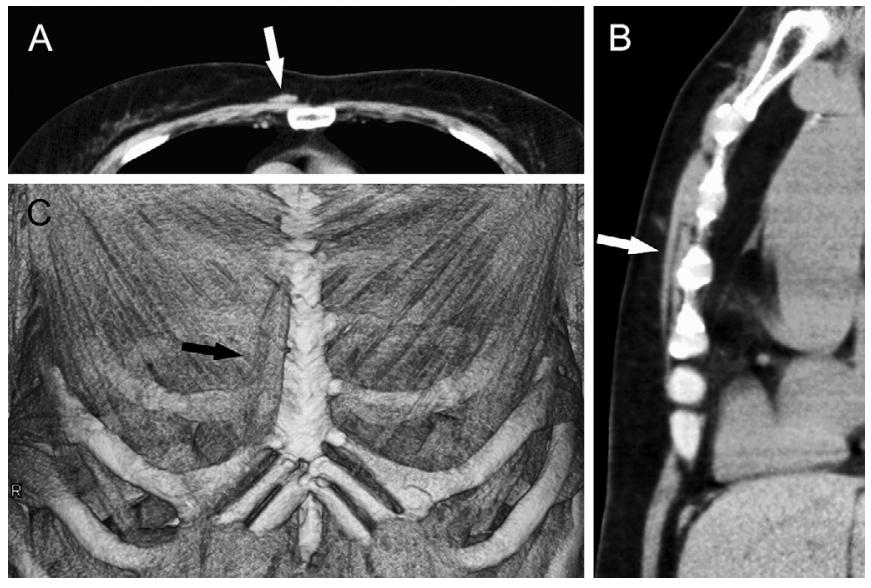

Figure 2. A 63-year-old woman with a sternalis muscle in the right anterior chest wall. A: A routine axial MDCT image shows a soft tissue mass (arrow), i.e., the sternalis muscle, which lies superficial to the right pectoralis major muscle. B: A postprocessed 2D-MPR image in the sagittal section reveals the corresponding soft tissue mass (arrow), which runs longitudinally in a craniocaudal direction. C: A 3D-VR image provides the entire configuration of the sternalis muscle, which has a fusiform shape with fascicle formation (arrow), thus reflecting a muscular architecture similar to that of other musculature in the chest wall.

3D-VR reconstruction images, each mass clearly demonstrated a fusiform configuration with fascicle formation and was located in the right paramedian position with a longitudinal course between the infraclavicular region and the caudal aspect of the sternum superficial to the pectoralis major muscle. These additional postprocessed MDCT images combined with routine axial images confirmed the morphological characteristics of the sternalis muscle and therefore the diagnosis was made with a high confidence level.

\section{Discussion}

In this case report, we demonstrated the utility and value of additional postprocessed 2D-MPR and 3D-VR images combined with routine axial images in the diagnosis of the sternalis muscle detected incidentally on routine chest MDCT images. To the best of our knowledge, this is the first report to emphasize assessment of the sternalis muscle using multiple postprocessed MDCT images.

The sternalis muscle is an uncommon muscular variant of the chest wall located in the parasternal region. On autopsy, the prevalence of this muscle varies according to ethnicity; for example, $4.1 \%-15.6 \%$ in the Japanese population, $1.0 \%$ $23.5 \%$ in the Asian population, $4.0 \%-8.0 \%$ in the Indian population, $1.9 \%-9.9 \%$ in the European and American populations and $4.2 \%-14.3 \%$ in the African population (7). On chest MDCT examinations conducted in living patients, the sternalis muscle is revealed in $6.2 \%-10.5 \%$ of cases $(2,3)$. Both sexes are affected with a slight dominancy in women, and the unilateral form is twice as common as the bilateral form $(2-4,7)$. Although the function and etiology of this 
muscle are unclear, the sternalis muscle is clinically important in some cases; for example, it may be used in breast reconstruction after mastectomy (8) or adapted as a pedicle flap or flap with microvascular anastomosis during plastic and reconstructive surgery of the head and neck region (9). This muscle can be detected incidentally on routine imaging examinations such as mammography $(4), \operatorname{MDCT}(2,3)$ and magnetic resonance imaging, which can result in diagnostic dilemmas because many anatomical textbooks do not describe this muscle, and most surgeons and radiologists are not familiar with this anatomical variation (10). For example, Bradley et al. (4) reported that the sternalis muscle is depicted as an irregular medial density on mammography mimicking a breast carcinoma, thus resulting in unnecessary surgical exploration. Therefore, obtaining knowledge and a precise assessment of this muscle is important for optimal patient management.

Rapid technological advances and the clinical introduction of MDCT, coupled with consecutive progression of postprocessing techniques, have shown MDCT to be a powerful and essential imaging tool in clinical practice. Such developments in MDCT can significantly assist in the diagnosis of and therapeutic planning for a variety of oncologic, vascular and musculoskeletal abnormalities $(5,11)$. Modern MDCT scanners are capable of obtaining wide coverage in the Zaxis with narrow collimation during short breath-holding, which facilitates construction of fine postprocessed MDCT images such as 2D-MPR and 3D-VR reconstruction with reduction of motion artifacts and respiratory misregistration. Such data acquisitions can demonstrate not only normal anatomical structures, but also pathological conditions from multidimensional views, even though nonenhanced MDCT images are acquired (12).

In both of our patients, additional postprocessed MDCT images combined with routine axial images fully revealed the longitudinal course and configuration of the sternalis muscle between the infraclavicular region to approximately the caudal aspect of the sternum at a glance. This met the following characteristics of this muscle, as described by Jelev et al. (7): (1) a location between the superficial fascia of the anterior thoracic region and the pectoral fascia; (2) an origin from the sternum or infraclavicular region; (3) insertion into the lower ribs, costal cartilages, aponeurosis of the external oblique abdominis muscle or the sheath of the rectus abdominis; and (4) innervation by the anterior thoracic (pectoral) and/or intercostal nerves. According to the classification proposed by Jelev et al. (7), both of our cases were type I1 (simple unilateral belly), which is the most common type. The use of additional postprocessed 2D-MPR and 3DVR images combined with routine axial MDCT images allows physicians and radiologists to detect and confirm the sternalis muscle easily and quickly with a high confidence level. Therefore, we emphasize the effectiveness of addi- tional postprocessed 2D-MPR and 3D-VR images combined with routine axial MDCT images in resolving the difficulty of diagnosing this uncommon muscular variant, although other complicated and less frequent types of sternalis muscles will be encountered (3). Moreover, if this muscle is occasionally detected on mammography with inconclusive findings, as Bradley et al. (4) reported, then we strongly recommend performing MDCT examinations and constructing postprocessed 2D-MPR and 3D-VR images simultaneously to differentiate other breast conditions, thereby reducing unnecessary interventions and establishing an accurate and timely diagnosis.

In conclusion, the sternalis muscle can be clearly demonstrated on additional postprocessed 2D-MPR and 3D-VR MDCT images, and these images combined with routine axial images strongly assist in diagnosing this uncommon muscular variant which is encountered incidentally on chest MDCT examinations.

\section{The authors state that they have no Conflict of Interest (COI).}

\section{References}

1. Saeed M, Murshid KR, Rufai AA, Elsayed SE, Sadiq MS. Sternalis. An anatomic variant of chest wall musculature. Saudi Med J 23: 1214-1221, 2002.

2. Young Lee B, Young Byun J, Hee Kim H, et al. The sternalis muscles: incidence and imaging findings on MDCT. J Thorac Imaging 21: 179-183, 2006.

3. Shiotani M, Higuchi T, Yoshimura N, et al. The sternalis muscle: radiologic findings on MDCT. Jpn J Radiol 2012. DOI: 10.1007/ s11604-012-0114-3

4. Bradley FM, Hoover HC, Hulka CA, et al. The sternalis muscle: an unusual normal finding seen on mammography. AJR Am J Roentgenol 166: 33-36, 1996.

5. Higashino T, Ohno Y, Takenaka D, et al. Thin-section multiplanar reformats from multidetector-row CT data: utility for assessment of regional tumor extent in non-small cell lung cancer. Eur J Radiol 56: 48-55, 2005.

6. Itoh S, Ikeda M, Ota T, Satake H, Takai K, Ishigaki T. Assessment of the pancreatic and intrapancreatic bile ducts using $0.5-\mathrm{mm}$ collimation and multiplanar reformatted images in multislice CT. Eur Radiol 13: 277-285, 2003.

7. Jelev L, Georgiev G, Surchev L. The sternalis muscle in the Bulgarian population: classification of sternales. J Anat 199: 359-363, 2001.

8. Schulman MR, Chun JK. The conjoined sternalis-pectoralis muscle flap in immediate tissue expander reconstruction after mastectomy. Ann Plast Surg 55: 672-675, 2005.

9. Vandeweyer E. The sternalis muscle in head and neck reconstruction. Plast Reconstr Surg 104: 1578-1579, 1999.

10. Bailey PM, Tzarnas CD. The sternalis muscle: a normal finding encountered during breast surgery. Plast Reconstr Surg 103: 1189$1190,1999$.

11. Saba L, Pascalis L, Mallarini G. Multi-detector-row CT of muscles with volume rendering technique. Panminerva Med 51: 43-49, 2009.

12. Johnson PT, Horton KM, Fishman EK. Noncontrast multidetector CT of the kidneys: utility of 2D MPR and 3D rendering to elucidate genitourinary pathology. Emerg Radiol 17: 329-333, 2010.

(C) 2013 The Japanese Society of Internal Medicine http://www.naika.or.jp/imonline/index.html 